\title{
REAL HILBERT ALGEBRAS WITH IDENTITY
}

\author{
M. F. SMILEY
}

Lars Ingelstam recently proved [2] that a real Hilbert algebra with identity is a division algebra and must, therefore, be isomorphic to the reals, complexes or real quaternions. This interesting result had been conjectured by I. Kaplansky. The purpose of this note is to offer a greatly simplified version of Ingelstam's proof. Our proof uses only very elementary facts about Banach algebras and is entirely selfcontained.

Let $A$ be a real Hilbert algebra with identity. That is, we assume that $A$ is a real Hilbert space with inner product $(x, y)$ which is also a Banach algebra with identity $e$ under the norm $\|x\|=(x, x)^{1 / 2}$ and, in addition, we have $\|e\|=1$.

In any Banach algebra $B$, an element $x$ in $B$ has an inverse in $B$ provided that $B$ has an identity $e$ and $\|e-x\|<1$. This remark leads to

Lemma 1. If $x$ is in $A$ and $(e, x) \neq 0$, then $x$ has an inverse in $A$.

Proof. We may assume that $(e, x)>0$ by replacing $x$ by $-x$ if necessary. There is, then, a positive real number $\alpha$ such that $\alpha^{2}\|x\|^{2}$ $<2(e, x)$. But this is equivalent to $\|e-\alpha x\|<1$; consequently $\alpha x$ has an inverse and so does $x$.

Lemma 2. If $x$ is in $A$ and $x$ has no inverse in $A$, then with $g(\alpha)$ $=(\exp \alpha x)-e$, we have $\|\exp \alpha x\|^{2}=1+\|g(\alpha)\|^{2}$ for all real $\alpha$.

Proof. Since $x$ has no inverse in $A, x^{n}$ has no inverse in $A$ for all positive integers $n$. By Lemma $1,\left(e, x^{n}\right)=0$ for all positive integers $n$. Considering the series for $\exp \alpha x$, we see that $(e, g(\alpha))=0$ for all real $\alpha$ and the lemma follows at once.

Lemma 3. If $x$ is in $A$ and $x$ has no inverse in $A$, then $\exp \alpha x=e$ for all real $\alpha \geqq 0$.

Proof. By Lemma 2, it is enough to prove that $\|\exp \alpha x\|=1$ for all real $\alpha \geqq 0$. Let $h(\alpha)=\log \|\exp \alpha x\|=(1 / 2) \log \left(1+\|g(\alpha)\|^{2}\right)$ for all real $\alpha \geqq 0$. Then $h(0)=0, h(\alpha) \geqq 0, h(\alpha+\beta) \leqq h(\alpha)+h(\beta)$, for all real $\alpha, \beta \geqq 0$. It is easy to show that $\|g(\alpha)\| \leqq \alpha((\exp \|x\|)-1)$ for $0<\alpha<1$, and it follows that

Received by the editors December 11, 1963.

1 Research partially supported by NSF Grant GP-1447. 
(1)

$$
\lim _{\alpha \rightarrow 0+} h(\alpha) / \alpha=0
$$

Suppose that $h(\gamma)>0$ for some real $\gamma \geqq 0$. Then $\gamma>0$ and $h(\gamma)$ $\leqq n h(\gamma / n)$ for every positive integer $n$. Consequently, if $\gamma_{n}=\gamma / n$, we have $h\left(\gamma_{n}\right) / \gamma_{n} \geqq h(\gamma) / \gamma>0$ for every positive integer $n$. This contradicts (1). Hence $h(\alpha)=0$ for all real $\alpha \geqq 0$ and the lemma is proved.

REMARK. Our proof of Lemma 3 uses a variant of Ingelstam's proof of his very interesting Lemma 1 of [1].

Lemma 4. If $x$ is in $A$ and $x$ has no inverse in $A$, then $x=0$.

Proof. Suppose $x \neq 0$. Then we may choose a real number $\alpha<1$ such that $0<\alpha<2 /\|x\|(\exp \|x\|)$. But we have exp $\alpha x=e$ by Lemma 3 and hence

$$
\|x\|=\left\|(1 / \alpha) \sum_{k=2}^{\infty} \alpha^{k} x^{k} / k !\right\| ! \leqq \alpha x \|^{2}(\exp \|x\|) / 2,
$$

a contradiction.

TheOREM. Every real Hilbert algebra with identity is a division algebra and is, therefore, isomorphic to the reals, complexes or real quaternions.

\section{REFERENCES}

1. L. Ingelstam, $A$ vertex property for Banach algebras with identity, Math. Scand. 11 (1962), 22-32. 796.

2. - Hilbert algebras with identity, Bull. Amer. Math. Soc. 69 (1963), 794-

University of California, Riverside 